\title{
Percepção da qualidade de vida de atletas femininas de voleibol escolar em diferentes categorias
}

\author{
Quality of life perception of female school volleyball \\ athletes in different categories
}

Rodrigo Lara Rother, ${ }^{1}$ Suélen da Silveira Machado Silva'

'Centro Universitário Univates, Lajeado, RS, Brasil.

Recebido em: 25/02/2017 / Aceito em: 24/05/2017 / Publicado em: 30/06/2017

rodrigorother@univates.br

\section{RESUMO}

Objetivo: analisar os níveis de Qualidade de Vida (QV) percebidos por atletas de voleibol, de três categorias distintas, compará-los quanto às dimensões física, social, psicológica e ambiental e avaliar sua satisfação, quanto ao treinamento que tem realizado. Método: a amostra foi constituída de 36 atletas, do sexo feminino, com idade entre 13 a 17 anos. Para a análise da QV foi utilizado o questionário WHOQOL-bref e para a satisfação com treinamento jogos, descanso e desempenho foi utilizado o questionário esportivo. Os resultados foram tabulados e submetidos a análise estatística, obtendo-se médias, desvio padrão, Anova e Teste $t$, adotando uma significância de $p<0,05$. Resultados: apontam para escores de QV geral nos valores de 79,2 para o Mirim/sub13, 67 para o Infantil/sub15 e 75 para o Infanto Juvenil/sub17. A média geral apresentada pelas atletas participantes foi de 74 pontos, o que a literatura classifica como "Boa". O domínio Social foi o que obteve maior pontuação (82, Muito Bom) e o domínio Psicológico menor (71, Bom). Considerações finais: a QV das atletas é considerada "Boa", com escores altos em todas as categorias, evidenciando a Mirim/sub13 como a melhor. Não houve alterações da QV das atletas relacionadas à carga de treinamento, mas sim ao aumento brusco originado na transição de uma categoria para outra.

Palavras-chave: Qualidade de Vida; Atletas; Adolescentes

\section{ABSTRACT}

Objective: to analyze the Quality of Life (OL) levels perceived by athletes of three different categories, comparing them in terms of physical, social, psychological and environmental dimensions and to evaluate their satisfaction with the training they have performed. Method: a sample consisting of 36 female athletes between 13 and 17 years old. For the OL analysis, the WOQOL-bref questionnaire was used and for the satisfaction with games, rest and performance training, the sports questionnaire was used. The results were tabulated and submitted to statistical analysis obtaining means, standard deviation, Anova and t Test, adopting a significance level of $p<0.05$. Results: the general QL score was 79.2 for Mirim/sub13, 67 for Infantile/ sub15 and 75 for infant juvenile/sub17. The overall mean presented by the participating athletes was 74 points, which the literature classifies as "good". The Social domains was the one obtained the highest score (82, Very good) and the Psychological minor domain (71, Good). Closing remarks: the QL of athletes is considered "good", with high scores in all categories showing Mirim/sub13 as the Best. There were no changes in the $Q L$ of the athletes related to the training load, but rather to the sudden increase in the transition from on category to another.

Keywords: Quality of life; Athletes; Adolescents. 


\section{INTRODUÇÃO}

Vem se tornando cada vez mais comum a análise dos aspectos que influenciam na Qualidade de Vida (OV) da população em geral. Porém, o número de pesquisas científicas ainda é restrito e voltado quase que exclusivamente a populações especiais, como obesos, portadores de necessidades especiais ou patologias, por exemplo.

Uma vida saudável e equilibrada deveria ser objetivo de todas as pessoas. A QV está relacionada a um contexto harmonioso que o indivíduo obtém no seu dia a dia, considerando os aspectos físicos, ambientais, psicológicos e sociais, visto que o grau de satisfação que o indivíduo possui diante da vida observa esses vários aspectos. ${ }^{1}$

Nenhuma análise sobre a satisfação individual, quanto à $\mathrm{OV}$ poderá ser desenvolvida sem uma contextualização da mesma, na vida coletiva. ${ }^{2}$ Partindo dessa concepção, encontram-se na literatura classificações em duas esferas: objetiva e subjetiva. A esfera objetiva refere-se à disposição de recursos para saúde, alimentação, moradia e demais pontos relacionados às condições materiais que estão ao alcance da população. Já, a subjetiva diz respeito à percepção de cada indivíduo sobre suas condições físicas, emocionais, sociais e ambientais, dando seu valor para tal. ${ }^{3}$

É importante frisar que, mesmo tendo características distintas, as duas esferas complementam-se e contribuem cada uma, da sua forma, para a compreensão da QV das pessoas. Porém, independentemente da esfera observada, do instrumento indicador utilizado ou do conceito adotado sobre QV, a mesma tem uma íntima relação com a prática de atividade física. ${ }^{4}$

A atividade física é uma forma de o ser humano adotar um estilo de vida mais saudável para si. A sociedade contemporânea a coloca como uma ponte segura para melhores condições de saúde. É uma função bastante ampla para atribuir-se a um único conceito, sintetizando a abrangência das inúmeras consequências sobre o organismo humano, como controle do estresse, prática antissedentária e também para fins estéticos ou de melhora de performance atlética. ${ }^{5}$

A atividade física habitual representa uma das importantes características do estilo de vida individual que pode afetar a saúde. Quando bem orientada, proporciona aos seus praticantes benefícios biológicos como, por exemplo, a melhora da qualidade do sono e a redução dos níveis de hipertensão, além de benefícios psicológicos como a sensação de bem-estar, a redução do nível de estresse e dos sintomas de depressão e ansiedade; e ainda benefícios sociais, tais como interação com outras pessoas e maior socialização. ${ }^{6}$

Dentre os tipos de atividades físicas possíveis, uma das mais praticadas, atualmente, é o esporte, que surgiu na sociedade no século $\mathrm{XIX}$, tornando-se rapidamente um fenômeno social e contando, a cada dia, com mais adeptos. O esporte tornou-se a expressão hegemônica da cultura corporal de movimento pois, de uma forma ou outra, faz parte da vida da maioria das pessoas em todo o mundo. ${ }^{7}$

Os esportes apresentam-se em uma grande diversidade de modalidades. Dentre estas, destaca-se o voleibol, que é um dos esportes que cresceu muito no Brasil, sendo classificado como o segundo espor- te preferido pelos brasileiros. ${ }^{8}$ Esta modalidade vem ganhando cada vez mais adeptos por ser um esporte de simples acesso, promovendo intervenções, quanto à cooperação, convivência, participação e inclusão, entre outros. Os praticantes encontram no voleibol, uma ocupação positiva do tempo livre, possibilidades de novas relações sociais, adoção de um estilo de vida mais saudável e melhora da QV. ${ }^{9}$

A partir do momento em que a prática do voleibol passa de um formato de lazer, lúdico e participativo, para um formato de competição e melhora da performance, acrescentam-se aí características físicas mais específicas para o praticante. Entre elas estão à exigência de melhora constante na técnica e particularidades psicológicas que possam suportar estresse, dor, lesões e pressão pela vitória. ${ }^{10}$

Quando este formato competitivo ocorre nas categorias de base, onde estão os atletas jovens e em formação, o cuidado e as atenções para com os efeitos do treinamento sobre a sua QV devem ser redobrados. Cada idade, dentro do voleibol, possui exigências particulares, que aumentam à medida que o atleta passa de uma categoria para outra superior. Essa passagem determina a utilização de diferentes métodos de preparação, como o aumento da carga de treinamento e níveis mais competitivos nos torneios e campeonatos em que compete.

Outro fator que exige atenção é o tempo que o jovem atleta destina para a prática da modalidade, algumas vezes abandonando outras atividades para dedicar-se exclusivamente ao voleibol. É necessário que os técnicos formadores atentem para além das questões técnicas da modalidade e se adequem às novas exigências de todos os aspectos que podem interferir na performance, inclusive os culturais. ${ }^{11}$ Além do que, cada atleta possui sua característica peculiar, mesmo sendo da mesma idade, essas características influenciam muito, quando relacionadas a um grande grupo, podendo ocasionar vários problemas de relacionamento intra e extra quadra e que podem interferir na performance..$^{12}$

Nesse sentido, proporcionar QV aos atletas significa interligar essa gama de fatores. Quando em equilíbrio, o atleta pode obter sua melhor performance e contribuir com um melhor desempenho para a equipe. Porém, vale ressaltar, que para cada indivíduo, a QV está vinculada a vários fatores distintos e pode ter um significado, da mesma forma, distinto.

Considerando o voleibol como um fenômeno esportivo nacional, popularmente praticado e que sua prática sistemática como atividade física pode interferir na QV dos atletas, ainda mais quando estão no seu período de formação, este estudo tem por objetivo analisar os níveis de QV percebidos por atletas de três categorias distintas, compará-los quanto às dimensões física, social, psicológica e ambiental e avaliar sua satisfação, quanto ao treinamento que tem realizado.

\section{MATERIAIS E MÉTODO}

Esta investigação possui um caráter quantitativo, o qual buscou mensurar e comparar os níveis de QV percebidos por 36 atletas de uma equipe de voleibol feminino escolar, pertencentes a três diferentes catego- 
rias de base, sendo estas mirim/sub-13 (n12), infantil/ sub-15 (n11) e infanto-juvenil/sub-17 (n13). A equipe pertence a uma escola que participa de competições como Campeonato Estudantil do Rio Grande do Sul (CERGS) e dos Jogos Escolares da Juventude (JEJ), principais competições escolares do estado e do país. As atletas treinam em um município do interior do RS e residem neste local, em cidades próximas ou em um apartamento alugado pela instituição a qual fazem parte. Todas as atletas estudam na mesma escola, onde possuem bolsas de estudos como um incentivo à prática da modalidade.

Para verificação dos níveis de QV, foi utilizado o questionário WHOQOL-bref, versão abreviada do instrumento validado pelo grupo de estudos em QV da Organização Mundial da Saúde (OMS). Como este instrumento tem aplicação elaborada para adultos e o público alvo do presente estudo são crianças e adolescentes, duas questões sofreram alterações em sua apresentação, mas mantendo o domínio original. As duas perguntas são as de número 18 e 21, as quais falam de "trabalho" e "vida sexual", sendo substituídas por "treinamento" e "vida afetiva". Já, para verificar a percepção das atletas para com os treinamentos, os jogos, os intervalos de descanso e a satisfação com o desempenho coletivo e individual, aplicou-se um "Questionário Esportivo". O mesmo foi elaborado pelos pesquisadores, composto por oito perguntas fechadas, com opções de resposta em escala, numeradas de um a cinco. Os dois questionários foram aplicados no inicio de uma sessão de treinamento, na quadra do ginásio da escola, onde as atletas treinam, em momento avaliado como mais oportuno, pelo técnico da equipe.

Os dados estão descritos por meio de média, desvio padrão e distribuição de frequência. A comparação entre as médias foi realizada por meio de Anova Oneway, com Post Hoc de Bonferroni, sendo analisados no software SPSS v. 20.0, com um nível de significância de $p<0,05$. O projeto de pesquisa que resultou neste estudo foi aprovado pelo Comitê de Ética em Pesquisa da Univates, sob parecer número 1.379.815.

\section{RESULTADOS}

Cada categoria que integra o corpus deste estudo realiza treinamentos semanais com cargas gradualmente aumentadas em cada categoria superior, sendo que as atletas permanecem dois anos em cada categoria. A Mirim/sub-13 treina com periodicidade de três sessões por semana (total de 6 horas semanais), a categoria Infantil/sub-15 cinco sessões por semana (11 horas) e a categoria Infanto-juvenil/sub-17 também cinco vezes por semana (13 horas).

A percepção das atletas sobre a quantidade de treinamentos e jogos, ao tempo de descanso e recuperação e a sua satisfação com o desempenho individual e da sua equipe podem ser observadas na Tabela 1.

Os dados da tabela 1 mostram que a maioria das atletas informou perceber como "na medida certa" a quantidade de tempo destinada para treinamentos e competições. Esta foi a resposta de $83 \%$ a $85 \%$ das atletas, independente da categoria a qual pertence. Da mesma forma, o tempo destinado a jogos e competições é considerado "na medida certa" por entre $73 \%$ e $77 \%$ das atletas no geral.

Quanto ao nível de exigência enfrentado nos treinamentos, constatou-se que as atletas percebem como "na medida certa", em $75 \%$ das respostas no Mirim, $82 \%$ no Infantil e $85 \%$ no Infanto. Já, sobre o nível de exigência enfrentado em jogos e competições, constatou-se que para $67 \%$ no Mirim, $91 \%$ no Infantil e $85 \%$ no Infanto também estão "na medida certa". Com relação ao Mirim, que apresentou o valor mais baixo

Tabela 1 - Respostas mais comuns apresentadas no Questionário Esportivo pelas atletas.

\begin{tabular}{|c|c|c|c|}
\hline Questões & Categorias & $\%$ das respostas & Respostas \\
\hline \multirow{3}{*}{$\begin{array}{l}\text { Quantidade de tempo que você destina a } \\
\text { treinamento da sua de equipe; }\end{array}$} & MIRIM & 83 & Na medida \\
\hline & INFANTIL & 85 & Na medida \\
\hline & INFANTO & 85 & Na medida \\
\hline \multirow{3}{*}{$\begin{array}{l}\text { Quantidade de tempo que você destina a } \\
\text { jogos e competições da sua equipe; }\end{array}$} & MIRIM & 75 & Na medida \\
\hline & INFANTIL & 73 & Na medida \\
\hline & INFANTO & 77 & Na medida \\
\hline \multirow{3}{*}{$\begin{array}{l}\text { Nível de exigência que você enfrenta nos } \\
\text { treino da sua equipe; }\end{array}$} & MIRIM & 75 & Na medida \\
\hline & INFANTIL & 82 & Na medida \\
\hline & INFANTO & 85 & Na medida \\
\hline \multirow{3}{*}{$\begin{array}{l}\text { Nível de exigência que você enfrenta nos } \\
\text { jogos e competições da sua equipe; }\end{array}$} & MIRIM & 67 & Na medida \\
\hline & INFANTIL & 91 & Na medida \\
\hline & INFANTO & 85 & Na medida \\
\hline \multirow{3}{*}{$\begin{array}{l}\text { Tempo de descanso e recuperação entre } \\
\text { os treinos semanais; }\end{array}$} & MIRIM & 74 & Mais do que gostaria \\
\hline & INFANTIL & 45,4 & Menos do que gostaria \\
\hline & INFANTO & 46 & Mais do que gostaria \\
\hline \multirow{3}{*}{$\begin{array}{l}\text { Tempo de descanso após jogos e com- } \\
\text { petições; }\end{array}$} & MIRIM & 100 & Na medida \\
\hline & INFANTIL & 64 & Na medida \\
\hline & INFANTO & 54 & Mais do que gostaria \\
\hline \multirow{3}{*}{$\begin{array}{l}\text { Rendimento geral da sua equipe nas com- } \\
\text { petições que participa; }\end{array}$} & MIRIM & 75 & Plenamente Satisfatório \\
\hline & INFANTIL & 69 & Plenamente Satisfatório \\
\hline & INFANTO & 69 & Satisfatório \\
\hline \multirow{3}{*}{$\begin{array}{l}\text { Seu rendimento individual nos treinos e } \\
\text { jogos; }\end{array}$} & MIRIM & 75 & Satisfatório \\
\hline & INFANTIL & 64 & Satisfatório \\
\hline & INFANTO & 69 & Satisfatório \\
\hline
\end{tabular}


nesta questão; os $33 \%$ restantes somaram-se entre "mais do que gostaria" e "acima da minha capacidade", demonstrando clara percepção de que os jogos oficiais são muito difíceis para elas.

Se a maioria das atletas se mostra satisfeita com os níveis de treinamento e competições enfrentados, o mesmo não pode ser dito em relação ao tempo disponível para descanso entre as sessões de treinamentos semanais. As atletas da categoria Infantil $(45,4 \%)$ e da Infanto (46\%) afirmam que o descanso é "menos do que gostariam". Já, a categoria Mirim parece ser a única em que a maioria das atletas acredita descansar "mais do que o desejado" entre as sessões de treinamento da semana $(75 \%)$.

Quando o tempo de descanso refere-se ao pós-jogos e competições, $100 \%$ das atletas do Mirim, $64 \%$ do Infantil $54 \%$ do Infanto afirmam ter "mais do que gostaria". Já, para $36 \%$ das atletas Infantil e $30 \%$ das Infanto, percebem estar "na medida certa".

Sobre a satisfação com o rendimento da equipe nos jogos e competições, $75 \%$ das atletas Mirim e $69 \%$ das Infanto acreditam que o rendimento geral está "satisfatório". Na categoria infantil 73\% responderam "plenamente satisfatório". Esta satisfação também é presente com relação ao desempenho individual nos jogos e competições, onde se constatou que 75\%,64\% e $69 \%$ das atletas das categorias Mirim, Infantil e Infanto responderam "satisfatório". O que diferencia a categoria Mirim das outras é que os $25 \%$ restantes responderam estar "muito satisfatório" ou "plenamente satisfatório", enquanto no Infantil e no Infanto, o percentual restante divide-se na metade em "pouco satisfatório" e "muito satisfatório".

Quanto aos níveis de QV apresentados pelas atletas de cada categoria, subdividida nos domínios Físico, Social, Psicológico e Ambiental, podem ser observados na tabela 2. Os mesmos evidenciam que a QV Geral da categoria Mirim foi a melhor, com média de 79,2 pontos, seguido da Infanto com 75 pontos. A categoria Infantil foi a que apresentou média inferior, com 67 pontos. Esse nível geral mais elevado no Mirim reflete-se quando são analisados separadamente cada um dos domínios da QV. As atletas desta categoria apresentam níveis superiores às outras nos aspectos Físicos, Psicológicos, Sociais e Ambientais.

O mesmo ocorre com a categoria Infanto, que fica em segundo lugar, em todos os domínios, com exceção do Psicológico, no qual obteve o mesmo resultado encontrado na categoria Infantil (69 pontos). Esta última obteve resultados inferiores às outras categorias em todos os domínios, salvo a igualdade citada acima.

Ao analisar a média individual de cada categoria por domínio, iniciando pelo Físico, constata-se superioridade da categoria Mirim sobre as outras, sendo classifica- da como "Muito Boa" quanto à sua QV $(81,2)$. No domínio Psicológico, ocorre o mesmo fenômeno, com 80 pontos.

No Social, que apresentou maior média entre os domínios, todas as categorias obtiveram pontuação alta. Embora o Infantil tenha classificação como "Boa" e as outras categorias como "Muito Boa", foram obtidos 79 pontos, somente um ponto abaixo de ser classificado num nível superior em QV.

Já, o domínio Ambiental evidenciou-se mais a inferioridade na pontuação da categoria Infantil, que obteve 69 pontos. O Mirim e o Infanto obtiveram 77 e 76,2 pontos neste domínio. Ao observarem-se os domínios de forma isolada, percebe-se uma evidência no Social, onde foram encontrados 82 pontos na média, sendo o mais elevado. Os domínios Físico $(73,3)$, Psicológico (71) e Ambiental (74), obtiveram médias muito próximas, apresentando diferença estatisticamente significativa $(p<0,05)$ para com o primeiro. Os resultados obtidos para o domínio Social o classificam como "Muito Bom", já os outros em um nível abaixo, ou seja, "Bom" somente.

\section{DISCUSSÃO E CONSIDERACִÕES FINAIS}

Os resultados obtidos no questionário esportivo apresentaram variáveis sobre a percepção das atletas quanto às quantidades de treinamento e jogos, quanto ao tempo de recuperação e a satisfação com seu desempenho, onde encontrou-se um padrão de respostas indicando níveis adequados de exigência, pouco tempo para descanso e satisfação com o rendimento individual e coletivo. Quanto à QV, embora os níveis resultantes fossem considerados "bons", podemos constatar que houve uma oscilação na QV entre as categorias. Houve diminuição do Mirim para o Infantil, mas posterior crescimento do Infantil para o Infanto. Como as cargas de treinamento do Mirim para o Infantil passam de seis horas semanais para onze, talvez essa mudança brusca possa ter afetado os níveis de QV. Já, quando a mudança foi do Infantil para o Infanto, houve aumento de tempo de treinamento em menor quantidade, facilitando a adaptação e observando-se aumento na QV na categoria superior.

Uma carga excessiva de treinamentos e competições combinada com insuficiente recuperação pode causar uma série de transtornos para a saúde como cansaço físico e mental, lesões, fragilidade muscular, dores e desconfortos físicos, resfriados e/ou reações alérgicas, entre outros, podendo prejudicar de alguma forma a QV. ${ }^{13}$

Estudo relata que os níveis de QV de atletas são semelhantes aos encontrados em uma população normal, negando assim que o esporte de alto rendimento

Tabela 2 - Média e desvio padrão dos escores da QV nas categorias Mirim ( $n=12)$, Infantil ( $n=11)$ e Infanto Juvenil $(n=13)$, e geral da equipe

\begin{tabular}{lcccc}
\hline Dimensões QV & Mirim & Infantil & Infanto & Geral \\
\hline Físico & $81,22 \pm 9,30^{\mathrm{a}}$ & $66,91 \pm 14,58^{\mathrm{b}}$ & $71,77 \pm 11,29^{\mathrm{ab}}$ & $73,43 \pm 12,92$ \\
Psicológico & $79,83 \pm 7,09^{\mathrm{a}}$ & $64,82 \pm 12,58^{\mathrm{b}}$ & $68,00 \pm 10,98^{\mathrm{b}}$ & $70,97 \pm 12,02$ \\
Social & $86,14 \pm 7,40^{\mathrm{a}}$ & $78,72 \pm 14,95^{\mathrm{a}}$ & $80,77 \pm 11,56^{\mathrm{a}}$ & 0,003 \\
Meio Ambiente & $76,53 \pm 8,80^{\mathrm{a}}$ & $68,55 \pm 6,17^{\mathrm{b}}$ & $76,23 \pm 8,68^{\mathrm{a}}$ & $73,94 \pm 11,69$ \\
\hline
\end{tabular}

$p<0,05$ indica diferenças significativas entre as médias das categorias; letras sobescritas diferentes indicam o local das diferenças (Post Hoc). 
proporcione escores menores do que os apresentados pela população em geral. ${ }^{14}$ Outro estudo ${ }^{15}$ conclui que atletas possuem percepções diferentes em relação à vida esportiva, na qual estão inseridos, obtendo diferentes significados para cada um, o que pode explicar, de certa forma, tanto a afirmação acima quanto a oscilação nos escores de QV, encontrados na tabela 2.

Mesmo que as atletas considerem as cargas de treinamento como estando na medida certa, percebem também que o descanso é reduzido, criando-se uma preocupação já evidenciada em estudos anteriores, que afirmam que atletas mulheres preocupam-se mais com fatores relacionados à estrutura organizacional no ambiente de treinamento/competição e intervalos adequados de descanso e recuperação, justificando a interferência na sua $Q V{ }^{16}$

Apesar do pouco tempo de recuperação entre treinamentos, constata-se que as atletas das três categorias classificam seu rendimento individual como satisfatório. Devido às características da modalidade, o atleta de voleibol acaba por criar adaptações fisiológicas no seu organismo para atender às necessidades da modalidade, permitindo-Ihe assim um melhor desempenho de sua função esportiva, dentro da equipe. ${ }^{17}$

As atletas da categoria Mirim, que obtiveram maiores escores de QV que as outras categorias, informaram ter mais descanso que o desejado. Mas, deve-se considerar que são as que apresentam menor carga de treinamento semanal, subentendendo assim estarem dispostas a treinar mais. Indicadores de QV são importantes para o desporto, podendo afetar o desempenho e influenciar os resultados obtidos em competições. ${ }^{18}$ Explica-se o Mirim como possível melhor QV, já que possuem cargas de treinamentos inferiores às outras categorias, maior tempo de descanso, menor pressão por resultados positivos, por parte da comissão técnica e por terem a família sempre mais próxima; característica das atletas com idades menores. ${ }^{19} \mathrm{~A}$ literatura reforça ainda a importância da família, dos amigos e dos primeiros professores ou treinadores no incentivo para a prática esportiva de jogadores de voleibol.

$\mathrm{Na}$ análise da categoria infantil, essa tem seu maior escore no domínio social. O fato das atletas aumentarem as exigências em todos os sentidos com relação à categoria inferior também leva esta a passar muito mais tempo com suas colegas de equipe, seja em treinamento ou viajando para competições. Sente no contato com a família e amigos como sendo seu "porto seguro" e se mantém em equilíbrio com isso. Nos estudos relacionados à performance, alguns autores demonstram que o sucesso de jovens talentos passa, na maioria das vezes, pelo apoio da família e amigos. ${ }^{20}$

Uma das conclusões possíveis a partir desses dados é que o domínio mais desenvolvido pelas atletas é o social. Um estudo que avaliou os níveis de QV de atletas que disputam a Superliga no Brasil descobriu que o domínio que predominou com maior pontuação foi o social. ${ }^{21}$ Outros estudos corroboram com esta informação, afirmando que uma vida voltada para a prática de exercícios físicos ou esportivos pode contribuir para um melhor convívio social. ${ }^{22}$

Apesar da predominância do domínio social, alterações em um ou mais domínios podem implicar em alterações na QV em geral. ${ }^{23}$ Não há nenhum aspecto ou domínio, nos dados aqui encontrados que demonstre uma diminuição na QV das atletas. Em um estudo que investigou a percepção da QV em atletas de voleibol em diferentes categorias e idades, constatou um alto nível de satisfação plena percebida em todas as equipes investigadas. ${ }^{24}$

A prática de alto rendimento não pode ser tida como essencialmente negativa à $\mathrm{QV}$ do atleta, assim como o esporte de lazer não pode ser tido como totalmente benéfico. ${ }^{25} \mathrm{O}$ significado da prática depende da adequação do sentido e da perspectiva em que o sujeito se coloca ou atua. ${ }^{26}$

Pode-se, portanto, dizer que QV é um processo instável, sujeito a mudanças rápidas e fortemente influenciado por ações do sujeito e do ambiente. Não apenas um estado físico puro objetivo que apresenta funções orgânicas intactas, mas considera também as dimensões subjetivas, individuais, psíquicas, mentais e sociais de cada pessoa. ${ }^{27}$ De forma geral, percebem-se nos resultados obtidos aqui o indicativo que atletas de voleibol feminino de base pertencentes a diferentes categorias apresentam escores de OV classificados como bons, apresentando valores de 79,2 para o Mirim/ sub13, 67 para o Infantil/sub15 e 75, para o Infanto Juvenil/sub17. Os níveis de QV geral apresentados pelas atletas participantes deste estudo obteve média de 74 pontos, o que a literatura classifica como sendo "Boa". ${ }^{28}$ Considerando que o limite do nível "bom" para o "muito bom" está em 81 pontos, este valor pode ser considerado alto.

O valor prático deste estudo possibilita uma orientação à comissão técnica das equipes de voleibol de base, quanto à graduação no aumento das cargas de treinamento para cada categoria. Como os resultados evidenciaram que não é a quantidade de treinamento, tampouco o aumento gradual das horas de treinamento que promovem aumento ou diminuição dos escores de QV, a atenção sobre a queda da QV deve voltar-se ao aumento brusco ou desproporcional nas horas de treinamento semanais. O presente estudo não buscou como objetivo analisar a relação entre desempenho e $\mathrm{QV}$, mas acredita-se que futuros estudos possam confirmar que há relação positiva e significativa entre os mesmos.

\section{REFERÊNCIAS}

1. Cheik NC, Reis IT, Heredia RAG, Ventura ML, Tufik S, Antunes HKM, Mello MT. Efeitos do exercício físico e da atividade física, na depressão e ansiedade em indivíduos idosos. Rev Bras Ci e Mov 2003;11(3):45-52.

2. Tubino MJG. Qualidade de Vida e sua complexidade. In: Moreira WW, Simões R (orgs.). Esporte como fator de Qualidade de Vida. Piracicaba: Editora Unimep, 2002. p. 263-268.

3. Gonçalves A, Vilarta R. Qualidade de vida: identidades e indicadores. In: Gonçalves A, Vilarta R. (orgs). Atividade física e qualidade de vida: explorando teoria e prática. Barueri, Manole. 2004.

4. Garcia R. Desporto, formação profissional e Qualidade de Vida. In: Moreira WW, Simões R (orgs.). Esporte como fator de Qualidade de Vida. Piracicaba: Editora UNIMEP, 2002, 163-72. 
5. Lovisolo H. Atividade física e saúde: uma agenda sociológica de pesquisa. In: Moreira WW, Simões R. (orgs.). Esporte como fator de Qualidade de Vida. Piracicaba: Editora UNIMEP, 2002, p. 277-296.

6. Lopes A, Pène P, Touitou Y. Sport and health. Bull Acad Natl Med 2009;193(2):415-29.

7. Bracht V. Sociologia Crítica do Esporte: Uma Introdução. 3. ed. Ijuí: Ed. Unijuí, 2005. 164 p.

8. Aranda L, Torga D, Fortes L, Bara FMG. Influência do treinamento de voleibol na composição corporal de atletas do gênero feminino durante uma temporada regular. Coleção Pesquisa em Educação Física. 2010.

9. Gonçalves KSM, Melo SLP, Pereira EGB. Análise da qualidade de vida de praticantes de voleibol de praça na zona oeste do município do Rio de Janeiro. Lecturas: Educación Física y Deportes 2009;132(14):45-56.

10. Berger BG, Pargman D, Weinberg RS. Foundations of exercise Psychology. Morgantown USA: Fitness Information Techonology, 2007.

11. Rother RL, Franzen C. A formação do atleta no contexto histórico da educação física e do treinamento esportivo no Brasil. Lecturas; Educacion Física y Deportes 2005;197(19): 34.

12. Organização Mundial da Saúde. The world health organization quality of life assessment (WHOQOL): Position paper from the world health organization. Social Science and Medicine 1995;41(10):403-9.

13. Costa LOP, Samulski DM. Processo de validação do questionário de estresse e recuperação para atletas (RESTO - Sport) na língua portuguesa. Revista Brasileira de Ciência e Moviment 2005;13(1):79-86.

14. Correia R, Tomazzoni F. Qualidade de vida de atletas de alto rendimento: uma analise do perfil das jogadoras de voleibol dos principais clubes universitários brasileiros. Faculdade de Educação Física-Unicamp, 2010. 94 p.

15. Hansel LG, Neuenfeldt DJ. A trajetória esportiva de dois ex-atletas iniciados precocemente na modalidade de basquetebol: da iniciação ao abandono. Lecturas en Educación Física y Deporte 2007;108(12):60-9.

16. Rother $R L$, Rempel C. Voleibol, treinamento e qualidade de vida de jovens atletas: reflexões teóricas. Lecturas en Educación Física y Deporte 2016;216(21):55-64.

17. Arruda M, Hespaniol JE. Fisiologia do Voleibol. São Paulo:
Phorte, 2008

18. Freitas CMSM, Farias JJM, Sandes JAB, Kucera CAC, Melo RR, Leão AC, Cunha A EV. Aspectos psicossociais que interferem no rendimento de modalidades desportivas coletivas. Revista Brasileira Cineantropometria e Desempenho Humano 2009;11(2):195-201.

19. Noce F, Samulski D. Análise do estresse psíquico em atacantes no voleibol de alto nível. Rev Paul Educ Fís 2002;16(2):113-29.

20. Moraes LC, Rabelo AS, Salmela JH. Papel dos pais no desenvolvimento de jovens futebolistas. Psicol Reflex Crit 2004. 17(2):211-22. doi: 10.1590/S010279722004000200009

21. Machi CL, Santos DS, Kemper G, Cordeiro MV, Oliveira AL. Analise de Qualidade de Vida das jogadoras de vôlei profissional da cidade de Brusque-SC. XIV INIC-Encontro Latino Americano de Iniciação Cientifica. UNIVAP 2010.

22. Noce F, Simim MAM, Mello MT. A percepção de qualidade de vida de pessoas portadoras de deficiência física pode ser influenciada pela prática de atividade física? Rev Bras Med Esporte 2009;15(3):174-8.

23. Pereira RJ, Cota RMM, Franceschini CC, Ribeiro RCL, Sampaio F, Priore SE. Contribuição dos domínios físico, social, psicologico e ambiental para a qualidade de vida global de idosos. Rev Psiquiatr Rio Gd Sul 2006;28(4):27-38.

24. Simões CSM. Analise das percepções da Qualidade de vida, do estresse e da recuperação de atletas de voleibol de diferentes categorias. Belo Horizonte/MG: Universidade Geral de Minas Gerais. Escolas de Educação Física, Fisioterapia e Terapia Ocupacional. Dissertação de Mestrado. 2011.

25. Villarta M, Gutierrez L, Monteiro MI. Qualidade de vida: evolução dos conceitos e práticas no século XXI. Ipes, 2010.

26. Marques RFR. Esporte e Qualidade de Vida: Reflexão Sociológica. Dissertação de mestrado. Faculdade de Educação Física da Universidade Estadual de Campinas, Campinas, 2007.

27. Weineck J. Atividade Física e Esporte: Para quê? Barueri: Manole, 2003.

28. Padrão MB. Avaliação da qualidade de vida de doadores vivos após o transplante renal utilizando os instrumentos SF - 36 e WHOQOL- bref. 2008. Dissertação Mestrado. Faculdade de Ciências Médicas, São Paulo/SP. 2008.

Como citar: ROTHER, Rodrigo Lara, et al. Percepção da qualidade de vida de atletas femininas de voleibol escolar em diferentes categorias. Cinergis, Santa Cruz do Sul, v. 18, n. 3, maio 2017. ISSN 2177-4005. Disponíve/ em: <https://online.unisc.br/seer/ index.php/cinergis/article/view/9145>. Acesso em: 22 jun. 2017. doi:http://dx.doi.org/10.17058/cinergis.v18i3.9145. 